\title{
Exploratory Investigation of Genetic Associations with Basal Cell Carcinoma Risk: Genome-Wide Association Study in Jeju Island, Korea
}

\author{
Byung Min Yun', Jung-Kook Song ${ }^{2 \#}$, Ji-Young Lee ${ }^{3 *}$
}

\begin{abstract}
Aim: Little is known about the genetic associations with Basal cell carcinoma (BCC) risk in non-Caucasian populations, in which BCC is rare, as in Korea. We here conducted a pilot genome-wide association study (GWAS) in 12 patients and 48 standard controls. Method: A total of 263,511 SNPs were analyzed with the Illumina HumanOmni1 Quad v1.0 DNA Analysis BeadChip for cases and Korean HapMap 570K for controls. Results: SNP-based analyses, based on the allele genetic model with adjustment for sex and age showed suggestive associations with $\mathrm{BCC}$ risk for $6 \mathrm{SNPs}$ with a $\mathrm{P}$-value $(\mathrm{P}<\mathbf{0 . 0 0 0 5})$. However, these associations were not statistically significant after Bonferroni correction: rs1040503, rs2216491, rs13407683, rs4751072, rs9891263, and rs1368474. In addition, results from gene-based analyses showed suggestive associations with BCC risk for 33 candidate genes with a $P$-value $(P<\mathbf{0 . 0 0 0 5})$. Consistent with previous $G W A S$ and replication studies in Caucasian populations, PADI6, RHOU and SLC45A2 were identified as having null associations with BCC (P> 0.05), likely due to the smaller sample size. Conclusions: Although this was a small-scale negative study, to our knowledge, we have conducted the first GWAS for BCC risk in an Asian population. Further large studies in non-Caucasian populations are required to achieve statistical significance and confirm these findings.
\end{abstract}

Keywords: Basal cell carcinoma - genome-wide association study - Korea

Asian Pac J Cancer Prev, 15 (17), 7443-7447

\section{Introduction}

Basal cell carcinoma (BCC), also called basalioma, basal cell epithelioma, rodent ulcer and Jacobs' ulcer, is the most common cancer among Caucasians (Madan, 2010). BCC can grow aggressively, causing extensive tissue destruction, if left untreated. Metastasis to lymph nodes, lung, liver and bone is very rare $(<0.1 \%$ ) (von Domarus et al., 1984; Elghissassi et al., 2009).

Recent insights into the molecular etiology and pathogenesis of BCC have uncovered environmental and other genetic factors that could account for the development of BCC. Genome-wide association studies (GWAS) have been used in the identification of loci associated with BCC in Caucasian populations. Recent meta-analyses of GWAS results on pigmentation and skin cancers have conclusively demonstrated and identified the genetic loci associated with skin cancer, mostly BCC in Caucasians (Gerstenblith et al., 2010). However, little is known about the genetic loci in non- Caucasian populations with lower BCC incidence.

Although $\mathrm{BCC}$ has become the most common type of skin cancer today, only 2,140 newly diagnosed cases were reported in 2011, from the Korean population of approximately 50 million people (KCCR, 2014). In the present study we conducted a GWAS, as an exploratory investigation, to identify the genetic association with BCC risk in Jeju Island, Korea.

\section{Materials and Methods}

\section{Subjects}

A total of 12 cases were recruited from patients who had been diagnosed with histologically confirmed BCC at the Jeju National University Hospital, from May 2010 to April 2011. The province of Jeju is an island located apart from the southwest corner of the Korean Peninsula. It's the number of population was 57 thousands in 2011. A total of 48 control subjects were obtained from Korean HapMap Project (http://sysbio.kribb.re.kr:8080/khapmap/ index.jsp), matched to cases by sex and age. The subject pools are shown in Table 1.

The GWAS of BCC protocol was approved by the Institutional Review Board of the Jeju National University

${ }^{1}$ Departments of Plastic and Reconstructive Surgery, ${ }^{2}$ Department of Preventive Medicine, School of Medicine, Jeju National University, Jeju, ${ }^{3}$ Cardiovascular Genome Center, Yonsei University College of Medicine, Yonsei University, Seoul, Korea ${ }^{\sharp}$ Co-first author*For correspondence: newwd7@gmail.com 
Hospital. Informed consent was obtained from all patients. The study was conducted according to the Declaration of Helsinki Principles.

\section{Genotyping}

Genotyping was performed on DNA extracted from 12 cases using the Illumina HumanOmni1 Quad v1.0 DNA Analysis Bead Chip array containing 1,140,419 SNP loci with a marker distance of $1.2 \mathrm{~kb}$ (median) and a call rate of 99.79\% (Illumina, Inc., San Diego, CA, USA). However, Korean HapMap 570K was used for 48 controls with a call rate of $99.53 \%$.

\section{Genomic DNA quantitation}

DNA samples were checked the quality using NanoDrop® ND-1000 UV-Vis Spectrophotometer. Then samples were electrophoresed on agarose gels and samples with intact genomic DNA showing no smearing on agarose gel electrophoresis were selected for experiment. Intact genomic DNA was diluted to $50 \mathrm{ng} / \mu$ l concentration based on Quant-iT Picogreen (Invitrogen) quantitation. Concentrations were adjusted based on these results. All prepared samples were hybridized to Illumina Infinium Human-1 microarrays according to the manufacturer's protocols.

\section{Sample amplification and hybridization for BeadChips}

The whole-genome amplification process requires 200 ng of input gDNA and creates a sufficient quantity of DNA (1000X amplification) to be used on a single BeadChip in the Infinium assay (Illumina MP1 and AMM). After amplification, the product is fragmented using a proprietary reagent (FRG), precipitated with 2-propanol (plus precipitating reagent; PM1), and resuspended in formamide-containing hybridization buffer (RA1). The DNA samples are denatured at $95^{\circ} \mathrm{C}$ for $20 \mathrm{~min}$, then placed in a humidified container for a minimum of 16 $\mathrm{h}$ at $48^{\circ} \mathrm{C}$ allowing SNP loci to hybridize to the $50 \mathrm{mer}$ capture probes.

\section{Single-base extension and staining on BeadChips}

Following hybridization, the BeadChip/Te-Flow chamber assembly was placed on the temperaturecontrolled Tecan Flowthrough Rack, and all subsequent washing, extension and staining were performed by addition of reagents to the Te-Flow chamber.

For the single-base extension (SBE) (Infinium II) assay, primers were extended with a polymerase and labeled nucleotide mix (TEM), and stained with repeated application of STM (staining reagent) and ASM (antistaining reagent). After staining was complete, the slides were washed with low salt wash buffer (PB1), immediately coated with $\mathrm{XC} 4$, and then imaged on the Illumina BeadArray Reader.

Imaging the BeadChip and data analysis

The Illumina BeadArray Reader is a two-color (543 $\mathrm{nm} / 643 \mathrm{~nm}$ ) confocal fluorescent scanner with $0.84-\mu \mathrm{m}$ pixel resolution. The scanner excites the fluorophors generated during signal amplification/staining of the allele-specific (one color) extension products on the BeadChips. The image intensities are extracted using
Illumina's BeadScan software. SNPs were called using Illumina's GenomeStudio V2009 (Genotyping Module 1.19) (Illumina, Inc.).

\section{Quality control}

The number of identical SNPs was 970,342 with Illumina HumanOmni1 Quad v1.0 from cases, 576,292 with Korean HapMap 570K from controls and 346,710 as the overlapping contents between them. About $36 \%$ $(124,744$ SNPs) of these SNPs were located in introns, $30 \%(104,444$ SNPs) in flanking 5'UTR, 30\% (102,710 SNPs) in flanking 3'UTR, and the rest were functionally located in coding, 3'UTR, 5'UTR, and UTR. Out of the initial full set of 346,710 SNPs, we discarded 8,091 SNPs in the case and 1,809 SNPs in the control with SNP-wised call rate $<95 \%, 564$ SNPs solely in the control with allele frequencies deviating from Hardy-Weinberg equilibrium (HWE) $(\mathrm{p}<10-6)$, and 59,925 SNPs in the case and 40,883 SNPs in the control with minor allele frequency $(\mathrm{MAF})<0.01$. Therefore, the final SNP set for the genetic association analyses contained 263,511 SNPs. The Results and Procedure of Quality Control (QC) in Subjects and SNPs on the GWAS are summarized in Table 2.

\section{Statistical analyses}

We calculated genotype frequencies for each individual polymorphism and evaluated Hardy-Weinberg equilibrium to check the data quality and genotype error using 'genetics' and 'dgc.genetics' packages in the R program. Fisher's exact test statistic was used to compare the observed numbers of each genotype with those expected following chi-square distribution with a one degree of freedom (Weir, 1996).

Association analysis was performed for 263,511 common SNPs that passed QC criteria for cases and

Table 1. The Subject Pools

\begin{tabular}{lcccc}
\hline & \multicolumn{2}{c}{ Cases } & \multicolumn{2}{c}{ Controls } \\
& Male & Female & Male & Female \\
\hline Number of subjects & 4 & 8 & 12 & 12 \\
Age (mean, yr.) & 63 & 62 & 60 & 62 \\
\hline
\end{tabular}

Table 2. The results and procedure of quality control (QC) in subjects and SNPs on the GWAS

\begin{tabular}{|c|c|c|}
\hline & $\begin{array}{c}\text { No. of cases } \\
\text { or SNPs } \\
\text { in case group }\end{array}$ & $\begin{array}{l}\text { No. of controls } \\
\text { or SNPs } \\
\text { in control group }\end{array}$ \\
\hline \multicolumn{3}{|l|}{$\overline{\mathrm{QC} \text { procedure in subjects }}$} \\
\hline Subjects at the start of QC & 12 & 90 \\
\hline \multicolumn{3}{|l|}{ Exclusion of subjects by } \\
\hline Gender inconsistency & 0 & 0 \\
\hline \multicolumn{3}{|c|}{ Low call rate (Averaged call rate) } \\
\hline & $0(99.79 \%)$ & $0(99.53 \%)$ \\
\hline Final subjects & 12 & 48 \\
\hline \multicolumn{3}{|l|}{ QC in SNPs } \\
\hline \multicolumn{3}{|c|}{ SNPs at the start of QC in each group } \\
\hline & 346,710 & 346,710 \\
\hline \multicolumn{3}{|l|}{ Exclusion of SNPs by } \\
\hline HWE $<10-6$ & & 564 \\
\hline Call rate $<95 \%$ & 8,091 & 1,809 \\
\hline MAF $<0.01$ & 59,925 & 40,883 \\
\hline Final SNPs in total subjects & 263,511 & \\
\hline
\end{tabular}


Table 3. Individual SNP-based Analyses: Associations between Skin Basal Cell Cancer and SNPs with a p-value $\left(<5 \times 10^{-4}\right)$ from a Genetic Model

\begin{tabular}{lcllr}
\hline SNP & Chromosome & Gene & Location & $*$ OR $(95 \%$ CI $)$ \\
\hline rs1040503 & 1 & ATP1B1 & intron & $9.82(3.12-30.90)$ \\
rs2216491 & 2 & LOC344371 & flanking_5UTR & $8.62(2.70-27.50)$ \\
rs13407683 & 2 & LOC344371 & flanking_5UTR & $8.62(2.70-27.50)$ \\
rs4751072 & 10 & MGMT & flanking_5UTR & $10.18(3.54-29.27)$ \\
rs9891263 & 17 & TOM1L1 & flanking_5UTR & $10.29(3.26-32.50)$ \\
rs1368474 & 19 & LPHN1 & flanking_5UTR & $1.7 \times 10^{-4}$ \\
*Allele OR adjusting for age and sex & & & $9.79(3.01-31.86)$ \\
\end{tabular}

Table 4. Individual Gene-based Analyses: Associations between Skin Basal Cell Cancer and SNPs with a p-value $\left(<5 \times 10^{-4}\right)$

\begin{tabular}{|c|c|c|c|c|c|c|c|c|}
\hline Gene & No. of SNPs per gene & flanking_5'UTR & 5'UTR & intron & coding & 3'UTR & flanking_3'UTR & p-value ${ }^{a}$ \\
\hline LOC727819 & 31 & 16 & 0 & 1 & 0 & 0 & 14 & $2.94 \times 10^{-10}$ \\
\hline ATOH1 & 33 & 9 & 0 & 0 & 0 & 0 & 24 & $3.32 \times 10^{-9}$ \\
\hline LOC644681 & 71 & 38 & 0 & 0 & 0 & 0 & 33 & $9.62 \times 10^{-9}$ \\
\hline C20orf 23 & 52 & 15 & 0 & 36 & 1 & 0 & 0 & $6.49 \times 10^{-8}$ \\
\hline LOC440337 & 43 & 4 & 0 & 0 & 2 & 0 & 37 & $3.63 \times 10^{-7}$ \\
\hline KRT8P21 & 30 & 15 & 0 & 0 & 0 & 0 & 15 & $5.89 \times 10^{-7}$ \\
\hline CYP3A7 & 11 & 0 & 0 & 7 & 0 & 1 & 3 & $9.98 \times 10^{-7}$ \\
\hline HLA-DQB1 & 48 & 43 & 0 & 0 & 0 & 2 & 3 & $1.31 \times 10^{-6}$ \\
\hline GPC6 & 156 & 28 & 0 & 126 & 1 & 0 & 1 & $2.48 \times 10^{-6}$ \\
\hline $\mathrm{ABCC} 2$ & 23 & 0 & 1 & 18 & 0 & 0 & 4 & $5.64 \times 10^{-6}$ \\
\hline FBN2 & 35 & 8 & 0 & 21 & 4 & 0 & 2 & $1.07 \times 10^{-5}$ \\
\hline LOC645174 & 36 & 16 & 0 & 0 & 0 & 0 & 20 & $1.39 \times 10^{-5}$ \\
\hline C10orf107 & 26 & 13 & 0 & 8 & 0 & 0 & 5 & $1.48 \times 10^{-5}$ \\
\hline XCL2 & 10 & 4 & 0 & 0 & 0 & 0 & 6 & $2.36 \times 10^{-5}$ \\
\hline C8orf34 & 95 & 8 & 1 & 47 & 1 & 0 & 38 & $2.41 \times 10^{-5}$ \\
\hline CALD1 & 29 & 7 & 0 & 20 & 1 & 0 & 1 & $3.48 \times 10^{-5}$ \\
\hline CDH13 & 430 & 71 & 0 & 358 & 0 & 0 & 1 & $3.79 \times 10^{-5}$ \\
\hline TYRP1 & 135 & 129 & 0 & 3 & 0 & 0 & 3 & $3.90 \times 10^{-5}$ \\
\hline LRP12 & 20 & 19 & 0 & 1 & 0 & 0 & 0 & $4.50 \times 10^{-5}$ \\
\hline C9orf66 & 12 & 7 & 0 & 0 & 1 & 0 & 4 & $5.91 \times 10^{-5}$ \\
\hline ELL2 & 12 & 2 & 0 & 8 & 0 & 0 & 2 & $5.91 \times 10^{-5}$ \\
\hline TANC1 & 26 & 4 & 0 & 21 & 0 & 1 & 0 & $6.25 \times 10^{-5}$ \\
\hline LOC728048 & 50 & 12 & 0 & 0 & 0 & 0 & 38 & $8.30 \times 10^{-5}$ \\
\hline TMEM26 & 22 & 3 & 0 & 3 & 0 & 1 & 15 & $8.40 \times 10^{-5}$ \\
\hline LOC132817 & 64 & 37 & 0 & 2 & 0 & 0 & 25 & $1.03 \times 10^{-4}$ \\
\hline CLCN4 & 23 & 0 & 0 & 12 & 0 & 3 & 8 & $1.12 \times 10^{-4}$ \\
\hline BARHL2 & 34 & 3 & 0 & 0 & 0 & 0 & 31 & $1.20 \times 10^{-4}$ \\
\hline TFEC & 34 & 3 & 0 & 7 & 0 & 1 & 23 & $1.20 \times 10^{-4}$ \\
\hline MAPRE2 & 19 & 5 & 0 & 8 & 0 & 1 & 5 & $1.49 \times 10^{-4}$ \\
\hline RNF130 & 19 & 1 & 0 & 13 & 0 & 0 & 5 & $1.49 \times 10^{-4}$ \\
\hline LOC344371 & 134 & 129 & 0 & 0 & 0 & 0 & 5 & $1.84 \times 10^{-4}$ \\
\hline $\mathrm{ABO}$ & 15 & 3 & 0 & 5 & 3 & 0 & 4 & $1.87 \times 10^{-4}$ \\
\hline PGRMC2 & 25 & 24 & 0 & 0 & 0 & 0 & 1 & $1.94 \times 10^{-4}$ \\
\hline
\end{tabular}

aFisher's exact test

controls under an allele genetic model (one degree of freedom) of logistic regression with adjustment for age and gender in 12 cases and 48 controls. And we controlled false discovery rate (FDR) to deal with multiple testing problem (Sorin, 2003). We adopted the conservative Bonferroni correction, which is the most commonly used method to adjust type 1 error, $\alpha$, treats each single-SNP test as an independent test. The Bonferroni threshold for significance of association was $1.89 \times 10^{-7}(\alpha=0.05 / 263,511)$.

\section{Results}

The results for SNP-based analyses through allele genetic model with adjustment for sex and age showed suggestive associations with BCC risk for 6 SNPs with a $\mathrm{p}$-value $\left(\mathrm{p}<5 \times 10^{-4}\right)$ in Table 3 . However, these were not statistically significant after Bonferroni correction: rs 1040503 (OR, 9.82;95\% CI, 3.12-30.90; $\mathrm{p}=9.4 \times 10^{-5}$ ), rs2216491 (A>G) (odds ratio (OR), 8.62; 95\% confidence interval(CI), 2.70-27.50; $\mathrm{p}=2.7 \times 10-4), \mathrm{rs} 13407683(\mathrm{~A}>\mathrm{G})$ (OR, 8.62;95\% CI, 2.70-27.50; $\mathrm{p}=2.7 \times 10-4)$, rs 4751072 (OR, 10.18;95\% CI, 3.54-29.27; $\mathrm{p}=1.7 \times 10-5$ ), rs9891263 (OR, 10.29;95\% CI, 3.26-32.50; $\mathrm{p}=7.0 \times 10-5)$ and rs1368474 (OR, 9.79; 95\% CI, 3.01-31.86; $\mathrm{p}=1.5 \times 10-4$ ). And the results for gene-based analyses showed suggestive associations with BCC risk for 33 suspected genes with a p-value $\left(\mathrm{p}<5 \times 10^{-4}\right)$ in Table 4.

\section{Discussion}

Using large populations with mostly Caucasian individuals, GWAS have produced sufficient evidence of the genetic associations with BCC risk (Stokowski et al., 2007; Sulem et al., 2007; Brown et al., 2008; Han et al., 2008; Stacey et al., 2008; Sulem et al., 2008; Falchi et al., 2009; Gudbjartsson et al., 2009; Nan et al., 2009a; Nan et 
al., 2009b; Rafnar et al., 2009; Stacey et al., 2009; Duffy et al., 2010). However, a paucity of studies based on data from non-Caucasian deals with the genetic associations with BCC risk (Cho et al., 2001; Kim et al., 2002; Kang et al., 2007). This may be because BCC is rare among African, Asians, and Hispanic populations (Yakubu et al., 1993; Diepgen et al., 2002). Although in Korea there is a very low incidence of BCC, we conducted a pilot GWAS in patients with histology-confirmed BCC in Jeju Island, Korea.

We performed a case control study comparing the frequency of SNP alleles between the Korean BCCs and the unaffected standard controls from the Korean HapMap Project. Since the controls were collected from the same ethnic background and geographical area as the case subjects, we could assume a good match between the genetic and environmental background of cases and controls (Lewis, 2002).

Single-SNP association tests were performed with logistic regression including sex and age as covariates. There were suggestive associations of the top six SNPs having a $\mathrm{p}$-value $\left(\mathrm{p}<5 \times 10^{-4}\right)$ with $\mathrm{BCC}$. These associations, however, were not significant after Bonferroni correction, our pilot GWAS was thus halted before the stage of replication. On the other hand, 33 putative genes showed suggestive associations with BCC risk from gene-based analyses. Although any of the ten genes identified as BCC risk factors by several previous GWAS and replication studies in Caucasian populations (PADI6, RHOU, SLC45A2, TERT/CLPTM1L, KLF14, CDKN2A/ B, TYR, KRT5, MC1R and ASIP) (Gerstenblith et al., 2010) were not included among our results of 33 genes, PADI6, RHOU and SLC45A2 were consistently identified notwithstanding that they had null associations with BCC ( $p>0.05)$, perhaps due to the smaller sample size.

The gene PADI6, peptidylarginine deiminase type six, carrying rs7538876 and the gene RHOU, ras homolog gene family member u, carrying rs801114 both are located in short arm 1p36 and long arm 1q42 chromosome 1, respectively (Stacey et al., 2008). The mechanisms by which these two loci increase the risk for BCC are unknown (Gerstenblith et al., 2010). The gene SLC45A2, solute carrier family 45 , member 2, carrying rs16891982 is located in short arm 5p13.3 chromosome 5. This is a potassium dependent sodium/calcium exchanger (Fernandez et al., 2008; Guedj et al., 2008). It has been reported that the SLC45A2 SNP rs 16891982 has a significant association with subjectively measured skin color in a population of European ancestry (Graf et al., 2007).

GWASs have investigated that BCC develops by at least two pathways: one pigmentation-dependent and one pigmentation-independent. SLC45A2, TYR, MC1R and ASIP suggest that BCC and melanoma share a common development pathway via pigmentation (Fernandez et al., 2008; Nan et al., 2008; Gudbjartsson et al., 2009). An alternative possibility is they do not themselves bring to BCC development but predispose to BCC. Since replication studies adjusted for pigmentation did not show consistent associations for some SNPs on TYRP1, TYR, and ASIP genes with BCC (Nan et al., 2009a; Duffy et al.,
2010), these genes should be investigated later whether subjects carrying these SNPs are associated with the risk of $\mathrm{BCC}$ via pigmentation alone or via pathways independent of pigmentation.

Epidemiological studies also suggested that BCC tumorigenesis may not unique genetic alteration. Disparities in clinic-pathologic characteristics were shown between sun-exposed head/neck BCC and lessexposed truncal BCC (Pelucchi et al., 2007; Betti et al., 2010). And a direct relation between UV exposure and BCC development was found for head/neck BCC only (Ramos et al., 2004). In addition, the lower mean age at occurrence of truncal BCC appears to support that BCC of sun-protected anatomic sites may related with decreased DNA repair capacity (Wei et al., 1993).

In the near future, surgical excision resulting in mutilating defects may no longer be the first treatment option for BCC. A good substitute for it will exist with the availability of limited primary excision followed by adjuvant therapy using an immunomodulator, a proapoptotic agent and/or a cell-signaling modulator (Tilli et al., 2005; Sekulic et al., 2012; Tang et al., 2012; Macha et al., 2013). This better and novel treatments and tailored drug therapy is regarded the most likely short-term benefit from genetic association studies (Hirschhorn et al., 2002).

We explored the genetic loci showing a putative association with BCC risk. To our knowledge, this is the first GWAS for BCC risk in Asian populations. However, the sample size was too small to achieve any statistical significance. Although we conducted a null study, this might contribute to avoid publication bias in metaanalyses later convincingly confirmed or refuted of all similar studies. Further larger studies in non-Caucasian populations are required to achieve statistical significance and to confirm the findings as independent replications (Zhang et al., 2012; Malik et al., 2014). And several important variables concerning gene should be considered such as family history of skin cancers and all other cancers, occupational sun exposure, smoking, and radiotherapy.

\section{Acknowledgements}

This study was supported by the research grant from the Jeju National University Hospital Research Fund of Jeju National University in 2010. We thank all of the patients for their participation.

\section{References}

Betti R, Menni S, Radaelli G, Bombonato C, Crosti C (2010). Micronodular basal cell carcinoma: A distinct subtype? Relationship with nodular and infiltrative basal cell carcinomas. J Dermatol, 37, 611-6.

Brown KM, MacGregor S, Montgomery GW, et al (2008). Common sequence variants on 20q11.22 confer melanoma susceptibility. Nat Genet, 40, 838-40.

Cho S, Hahm JH, Hong YS (2001). Analysis of p53 and BAX mutations, loss of heterozygosity, p53 and BLC2 expression and apoptosis in basal cell carcinoma in Korean patients. $\mathrm{Br}$ J Dermatol, 144, 841-8.

Diepgen TL, Mahler V (2002). The epidemiology of skin cancer. Br J Dermatol, 146, 1-6. 
Duffy DL, Zhao ZZ, Sturm RA, et al (2010). Multiple pigmentation gene polymorphisms account for a substantial proportion of risk of cutaneous malignant melanoma. J Invest Dermatol, 130, 520-8.

Elghissassi I, Mikou A, Inrhaoun H, et al (2009). Metastatic basal cell carcinoma to the bone and bone marrow. Int $J$ Dermatol, 48, 481-3

Falchi M, Bataille V, Hayward NK, et al (2009). Genome-wide association study identifies variants at $9 \mathrm{p} 21$ and $22 \mathrm{q} 13$ associated with development of cutaneous nevi. Nat Genet, 41, 915-8.

Fernandez LP, Milne RL, Pita G, et al (2008). SLC45A2: a novel malignant melanoma- associated gene. Hum Mutat, 29, 1161-7.

Gerstenblith MR, Shi J, Landi MT (2010). Genome-wide association studies of pigmentation and skin cancer: a review and meta-analysis. Pigment Cell Melanoma Res, 23, 587-606.

Graf J, Hodgson R, van Daal A (2005). Single nucleotide polymorphisms in the MATP gene are associated with normal human pigmentation variation. Hum Mutat, 25, 278-84.

Gudbjartsson DF, Sulem P, Stacey SN, et al (2009). ASIP and TYR pigmentation variants associate with cutaneous melanoma and basal cell carcinoma. Nat Genet, 40, 886-91.

Guedj M, Bourillon A, Combadieres C, et al (2008). Variants of the MATPSLC45A2 gene are protective for melanoma in the French population. Hum Mutat, 29, 1154-60.

Han J, Kraft P, Nan H, et al (2008). A genome-wide association study identifies novel alleles associated with hair color and skin pigmentation. PLoS Genet, 4, e1000074.

Hirschhorn JN, Lohmueller K, Byrne E, Hirschhorn K (2002). A comprehensive review of genetic association studies. Genet Med, 4, 45-61.

Kang SY, Lee KG, Lee W, et al (2007). Polymorphisms in the DNA repair gene XRCC1 associated with basal cell carcinoma and squamous cell carcinoma of the skin in a Korean population. Cancer Sci, 98, 716-20.

Kim MY,Park HJ, Baek SC, Byun DG, Houh D (2002). Mutations of the p53 and PTCH gene in basal cell carcinomas: UV mutation signature and strand bias. J Dematol Sci, 29, 1-9.

Korea Central Cancer Registry, the Korean Ministry of Health and Welfare. Unpublished data, 2014

Macha MA, Batra SK, Ganti AK (2013). Profile of vismodegib and its potential in the treatment of advanced basal cell carcinoma. Cancer Manag Res, 5, 197-203.

Madan V, Lear JT, Szeimies RM (2010). Non-melanoma skin cancer. The Lancet, 375, 673-85.

Malik MA, Umar M, Gupta U, Zargar SA, Mittal B (2014). Phospholipase C epsilon 1 (PLCE1 rs2274223A $>$ G, rs3765524C $>\mathrm{T}$ and rs7922612C $>\mathrm{T}$ ) polymorphisms and esophageal cancer risk in the Kashmir Valley. Asian Pac J Cancer Prev, 15, 4319-23.

Nan H, Kraft P, Hunter DJ, Han J (2009a). Genetic variants in pigmentation genes, pigmentary phentoypes and risk of skin cancer in Caucasians. Int J Cancer, 125, 909-17.

Nan H, Kraft P, Qureshi AA, et al (2009b). Genome-wide association study of tanning phenotype in a population of European ancestry. J Invest Dermatol, 129, 2250-7.

Pelucchi C, Di Landro A, Naldi L, La Vecchia C (2007). Risk factors for histological types and anatomic sites of cutaneous basal-cell carcinoma: An Italian case-control study. J Invest Dermatol, 127, 935-44.

Rafnar T, Sulem P, Stacey SN, et al (2009). Sequence variants at the TER-CLPTM1L locus associate with many cancer types. Nat Genet, 41, 221-7.

Ramos J, Villa J, Ruiz A, Armstrong R, Matta J (2004). UV dose determines key characteristics of nonmelanoma skin cancer.
Cancer Epidemiol Biomarkers Prev, 13, 2006-11.

Sekulic A, Migden MR, Oro AE, et al (2012). Efficacy and safety of vismodegib in advanced basal-cell carcinoma. $N$ Engl $J$ Med, 366, 2171-9.

Sorin D (2003). Data Analysis Tools For DNA Microarrays. U.S., CHAPMAN\&HALL/CRC.

Stacey, SN, Gudbjartsson DF, Sulem P, et al (2008). Common variants on $1 \mathrm{p} 36$ and $1 \mathrm{q} 42$ are associated with cutaneous basal cell carcinoma but not with melanoma or pigmentation traits. Nat Genet, 40, 1313-8.

Stacey, SN, Sulem P, Masson G, et al (2009). New common variants affecting susceptibility to basal cell carcinoma. Nat Genet, 41, 909-14.

Stokowski RP, Pant PVK, Dadd T, et al (2007). A genomewide association study of skin pigmentation in a South Asian population. Am J Hum Genet, 81, 1119-32.

Sulem P, Gudbjartsson DF, Stacey SN, et al (2007). Genetic determinants of hair, eye and skin pigmentation in Europeans. Nat Genet, 39, 1443-52.

Sulem P, Gudbjartsson DF, Stacey SN, et al (2008). Two newly identified genetic determinants of pigmentation in Europeans. Nat Genet, 40, 835-7.

Tang JY, Mackay-Wiggan JM, Aszterbaum M, et al (2012). Inhibiting the hedgehog pathway in patients with the basalcell nevus syndrome. $N$ Engl J Med, 366, 2180-8.

Tilli C, von Steensel M, Krekels G, Neumann H, Ramaekers FCS (2005). Molecular aetiology and pathogenesis of basal cell carcinoma. Br J Dermatol, 152, 1108-24.

von Domarus H, Stevens PJ (1984). Metastatic basal cell carcinoma: report of five cases and review of 170 cases in the literature. J Am Acad Dermatol, 10, 1043-60.

Wei Q, Matanoski GM, Farmer ER, Hedayati MA, Grossman L (1993). DNA repair and aging in basal cell carcinoma: a molecular epidemiology study. Proc Natl Acad Sci USA, 90, 1614-8.

Weir BS (1996). Genetic Data Analysis II: Methods for discrete population genetic data. Sunderland, MA, Sinauer Associates.

Yakubu A, Mabogunje OA (1993). Skin cancer in African albinos. Acta Oncol, 32, 621-2.

Zhang YR, Xu Y, Yang K, et al (2012). Association of six susceptibility loci with prostate cancer in northern Chinese men. Asian Pac J Cancer Prev, 13, 6273-6. 